\title{
Competencies of College Faculty Members of the Mindanao State University Tawi-Tawi College of Technology and Oceanography (Msu-Tcto): Their Implication to Teaching and Learning Efficiencies
}

\author{
Charity Joy B. Hashim ${ }^{1}$ \\ ${ }^{1}$ Mindanao State University-Tawi-Tawi, Bongao, Tawi-Tawi, Philippines
}

\begin{abstract}
Teaching has been described as the process of imparting knowledge and unifying process that affects almost every phase of growth, thinking, and personal and social development of the learners. It emphasizes the development of integrated personality of the child/learner - his abilities, habits, knowledge, skills, and attitudes. In other words, effective teaching and learning are the results of the integrated personality of the teacher. Thus, it is imperative that the teacher must be competent enough to achieve teaching effectiveness.
\end{abstract}

Keywords: Competencies, Teaching and Learning Efficiencies

\section{Introduction}

Teaching has been described as the process of imparting knowledge and unifying process that affects almost every phase of growth, thinking, and personal and social development of the learners. It emphasizes the development of integrated personality of the child/learner - his abilities, habits, knowledge, skills, and attitudes. In other words, effective teaching and learning are the results of the integrated personality of the teacher. Thus, it is imperative that the teacher must be competent enough to achieve teaching effectiveness.

Teacher competencies are defined as "the set of knowledge, skills, and experience necessary for future, which manifests in activities" Gupta, R. C (1976) define competencies as "knowledge, skills, attitudes, values, motivations and beliefs people need in order to be successful in a job".

This paper aims to discuss and identify the general framework of teachers' competencies. Among college faculty members of MSU-TCTO and how they relate to teaching and learning efficiencies. Teachers' competencies affect their values, behaviors, communication, aims, and practices in school and also they support professional development and curricular studies. Thus, the discussion on teachers' competencies to improve the teaching-learning process in school is of great importance.

\section{Problem Statements}

1. What is the socio-demographic profile of the respondents in terms of:

a. Gender

b. Civil Status

c. Work Status

d. Length of Service

e. Field of Specialization

f. Scholarship Enjoyed

g. Highest Educational Attainment and

h. Academic Awards? 
2. What is the level of faculty efficiency as measured by Teaching Efficiency Rating?

3. Is there a significant relationship between the socio-demographic profile and their competencies?

4. Is there significant difference in the ratings of the faculty based on the TER?

\section{Methods}

This research utilizes the survey approach of research using descriptive method. It was constituted of College faculty members of MSU-TCTO with a total of fifty two (52) coming from the different colleges, regardless of the field of specialization and length of service. Teaching Efficiency Rating (TER) result was used by the researcher in identifying their Teaching Competency.

The data for this study were the computerized Teaching Efficiency Rating (TER) of the faculty members, Academic year 2014-2015. Data were taken from the office of the Vice Chancellor for Academic Affairs. These were supplemented by the respondents' profile.

\section{Results}

Distribution of Respondents by Gender of the MSU-TCTO

\begin{tabular}{|c|c|c|}
\hline Gender Respondents & Frequency & Percent \\
\hline Male & 26 & 50.0 \\
\hline Female & 26 & 50.0 \\
\hline Total & 5.2 & 100.0 \\
\hline
\end{tabular}

Distribution of Respondents by Martial Status of the MSU-TCTO

\begin{tabular}{|c|c|c|}
\hline Marital Status & Frequency & Percent \\
\hline Single & 14 & 26.9 \\
\hline Married & 37 & 71.2 \\
\hline Widow/Widower & 1 & 1.9 \\
\hline Total & 52 & 100.0 \\
\hline
\end{tabular}

Distribution of Respondents by Appointment Status of the MSU-TCTO

\begin{tabular}{|c|c|c|}
\hline Work Appointment Status & Frequency & Percent \\
\hline Contract of Service & 8 & 15.4 \\
\hline Regular/Permanent & 44 & 84.6 \\
\hline Total & 52 & 100.0 \\
\hline
\end{tabular}

Distribution of the Respondents by Length of service in Years in MSU-TCTO

\begin{tabular}{|c|c|c|}
\hline Length of Service & Frequency & Percent \\
\hline $1-5$ years & 13 & 25.0 \\
\hline 6-10 years & 5 & 9.6 \\
\hline $11-15$ years & 5 & 9.6 \\
\hline 16-20 years & 11 & 21.2 \\
\hline 21-25 years & 5 & 9.6 \\
\hline 26 and more years & 13 & 25.0 \\
\hline Total & 52 & 100.0 \\
\hline
\end{tabular}


Distribution of Respondents by Field of Specialization in the different Department Colleges of MSU-TCTO

\begin{tabular}{|c|c|c|}
\hline Field of Specialization & Frequency & Percent \\
\hline Math/Stat & 8 & 15.4 \\
\hline Language & 13 & 25.0 \\
\hline Social Sciences & 7 & 13.5 \\
\hline Sciences (Physical, Natural, etc) & 6 & 11.5 \\
\hline Education & 3 & 5.8 \\
\hline ICT & 3 & 5.8 \\
\hline Sports & 3 & 5.8 \\
\hline Others & 2 & 3.8 \\
\hline Teaching Arabic & 1 & 1.9 \\
\hline Marine Biology & 3 & 5.8 \\
\hline Aquaculture & 1 & 1.9 \\
\hline Coastal Resource Mgnt & 1 & 1.9 \\
\hline Computer Education & 1 & 1.9 \\
\hline Total & 52 & 100.0 \\
\hline
\end{tabular}

Distribution of Respondents by Scholarship Enjoyed as Faculty Grantee

\begin{tabular}{|c|c|c|}
\hline Scholarship Enjoyed & Frequency & Percent \\
\hline None & 31 & 59.6 \\
\hline APDP & 13 & 25.0 \\
\hline CHEDPDF & 6 & 11.5 \\
\hline SSST & 1 & 1.9 \\
\hline DOST & 1 & 1.9 \\
\hline Total & 52 & 100.0 \\
\hline
\end{tabular}

Distributions of respondents by Highest Educational Attainment

\begin{tabular}{|c|c|c|}
\hline Highest Educational Attainment & Frequency & Percent \\
\hline Baccalaureate Degree & 14 & 26.9 \\
\hline Units in Master's Degree & 10 & 19.2 \\
\hline Masteral Degree & 22 & 42.3 \\
\hline Ph.D./Ed.D./DPA & 6 & 11.5 \\
\hline Total & 52 & 100.0 \\
\hline
\end{tabular}

Distribution of Respondents by Honor/Awards as they obtained their Baccalaureate Degree

\begin{tabular}{|c|c|c|}
\hline Honors/Awards & Frequency & Percent \\
\hline None & 30 & 57.7 \\
\hline Cum Laude & 15 & 28.8 \\
\hline Magna Cum Laude & 7 & 13.5 \\
\hline Total & 52 & 100.0 \\
\hline
\end{tabular}

Distribution of Respondents by Other Awards as They Obtained their Highest Educational Attainment

\begin{tabular}{|c|c|c|}
\hline Other_Awards & Frequency & Percent \\
\hline None & 37 & 71.2 \\
\hline Academic Excellence & 5 & 9.6 \\
\hline DOST Excellence Award & 1 & 1.9 \\
\hline Others & 8 & 15.4 \\
\hline LET Topnotcher & 1 & 1.9 \\
\hline Total & 52 & 100.0 \\
\hline
\end{tabular}


What is the level of faculty efficiency as measured by Teaching Efficiency Rating?

The Teaching Efficiency Ratings of the Respondents by Student, Peer and Superior in MSU-TCTO

\begin{tabular}{|c|c|c|c|}
\hline Ratings & Mean & Std. Deviation & N \\
\hline Rater, stud & 38.1325 & 1.32401 & 52 \\
\hline Rater_peer & 28.9052 & 0.65693 & 52 \\
\hline Rater_sup & 27.8779 & 1.94183 & 52 \\
\hline Total & 94.8960 & 2.33554 & 52 \\
\hline
\end{tabular}

Is there a significant relationship between the socio-demographic profile and their competencies?

Summary of the Correlation Coefficient of the Relationship of TER and the Demographic Profile of the Respondents - Correlations

\begin{tabular}{|c|c|c|c|c|c|c|c|c|c|c|}
\hline & & Gender & $\begin{array}{l}\text { Marital } \\
\text { Status }\end{array}$ & $\begin{array}{l}\text { Work } \\
\text { Status }\end{array}$ & $\begin{array}{l}\text { Service } \\
\text { length }\end{array}$ & FOS & $\begin{array}{c}\text { Scho } \\
\text { Enjoyed }\end{array}$ & $\begin{array}{c}\mathrm{HE} \\
\text { Attain }\end{array}$ & $\begin{array}{c}\text { Baccal } \\
\text { Awards }\end{array}$ & $\begin{array}{l}\text { Other__ } \\
\text { Award }\end{array}$ \\
\hline \multirow[t]{2}{*}{ Gender } & $\begin{array}{l}\text { Correlation } \\
\text { Value }\end{array}$ & 1 & .040 & -.213 & -.071 & -.102 & -.098 & -.126 & .080 & .000 \\
\hline & $\begin{array}{l}\text { Sig. (2- } \\
\text { tailed) }\end{array}$ & & .776 & .129 & .619 & .472 & .489 & .372 & .571 & 1.000 \\
\hline \multirow[t]{2}{*}{ Marital Status } & $\begin{array}{l}\text { Correlation } \\
\text { Value }\end{array}$ & .040 & 1 & .561 & .515 & .008 & -.031 & .615 & -.099 & .192 \\
\hline & $\begin{array}{l}\text { Sig. (2- } \\
\text { tailed) }\end{array}$ & .776 & & .000 & .000 & .953 & .828 & .000 & .487 & .173 \\
\hline \multirow[t]{2}{*}{ Marital Status } & $\begin{array}{l}\text { Correlation } \\
\text { Value }\end{array}$ & -.213 & .561 & 1 & .544 & .368 & .004 & .482 & -.188 & .096 \\
\hline & $\begin{array}{l}\text { Sig. (2- } \\
\text { tailed) }\end{array}$ & .129 & .000 & & .000 & .007 & .977 & .000 & .181 & .499 \\
\hline \multirow[t]{2}{*}{ Service length } & $\begin{array}{l}\text { Correlation } \\
\text { Value }\end{array}$ & $\begin{array}{l}.071 \\
\end{array}$ & .515 & .544 & 1 & .385 & .191 & .510 & $\begin{array}{l}.354 \\
\end{array}$ & .144 \\
\hline & $\begin{array}{l}\text { Sig. (2- } \\
\text { tailed) }\end{array}$ & .619 & .000 & .000 & & .005 & .174 & .000 & .010 & .308 \\
\hline \multirow[t]{2}{*}{ FOS } & $\begin{array}{l}\text { Correlation } \\
\text { Value }\end{array}$ & -.102 & .008 & .368 & .385 & 1 & .242 & -.015 & -.293 & .068 \\
\hline & $\begin{array}{l}\text { Sig. (2- } \\
\text { tailed) }\end{array}$ & .472 & .953 & .007 & .005 & & .083 & .914 & .035 & .630 \\
\hline \multirow[t]{2}{*}{ Scho Enjoyed } & $\begin{array}{l}\text { Correlation } \\
\text { Value }\end{array}$ & -.098 & -.031 & .004 & .191 & .242 & 1 & .169 & -.011 & .291 \\
\hline & $\begin{array}{l}\text { Sig. (2- } \\
\text { tailed) }\end{array}$ & .489 & .828 & .977 & .174 & .083 & & .231 & .938 & .036 \\
\hline \multirow[t]{2}{*}{ HE Attain } & $\begin{array}{l}\text { Correlation } \\
\text { Value }\end{array}$ & -.126 & .615 & .482 & .510 & -.015 & .169 & 1 & .077 & .156 \\
\hline & $\begin{array}{l}\text { Sig. (2- } \\
\text { tailed) }\end{array}$ & .372 & .000 & .000 & .000 & .914 & .231 & & .587 & .270 \\
\hline \multirow[t]{2}{*}{$\begin{array}{l}\text { Baccal } \\
\text { Awards }\end{array}$} & $\begin{array}{l}\text { Correlation } \\
\text { Value }\end{array}$ & .080 & $\begin{array}{l}.099 \\
\end{array}$ & -.188 & -.354 & .293 & $\begin{array}{l}-011 \\
\end{array}$ & .077 & 1 & .031 \\
\hline & $\begin{array}{l}\text { Sig. (2- } \\
\text { tailed) }\end{array}$ & .571 & .487 & .181 & .010 & .035 & .938 & .587 & & .828 \\
\hline \multirow[t]{2}{*}{ Other Award } & $\begin{array}{l}\text { Correlation } \\
\text { Value }\end{array}$ & .000 & .192 & .096 & .144 & .068 & .291 & .156 & .031 & 1 \\
\hline & $\begin{array}{l}\text { Sig. (2- } \\
\text { tailed) }\end{array}$ & 1.000 & .173 & .499 & .308 & .630 & .036 & .270 & .828 & \\
\hline \multirow[t]{2}{*}{ Rater_stud } & $\begin{array}{l}\text { Correlation } \\
\text { Value }\end{array}$ & .040 & -.066 & -.119 & .094 & -.055 & .050 & .029 & .121 & -.145 \\
\hline & $\begin{array}{l}\text { Sig. (2- } \\
\text { tailed) }\end{array}$ & .778 & .641 & .402 & .506 & .699 & .724 & .837 & .394 & .305 \\
\hline \multirow[t]{2}{*}{ Rater_peer } & $\begin{array}{l}\text { Correlation } \\
\text { Value }\end{array}$ & .024 & -.099 & -.070 & -.044 & .341 & -.130 & -.024 & -.023 & .022 \\
\hline & $\begin{array}{l}\text { Sig. (2- } \\
\text { tailed) }\end{array}$ & .866 & .485 & .620 & .756 & .013 & .356 & .866 & .873 & .879 \\
\hline \multirow[t]{2}{*}{ Rater_sup } & $\begin{array}{l}\text { Correlation } \\
\text { Value }\end{array}$ & .019 & .158 & .480 & .266 & .162 & .048 & .067 & $\begin{array}{l}-.078 \\
\end{array}$ & $\begin{array}{l}-.038 \\
\end{array}$ \\
\hline & $\begin{array}{l}\text { Sig. (2- } \\
\text { tailed) }\end{array}$ & .895 & .263 & .000 & .057 & .252 & .737 & .637 & .581 & .787 \\
\hline \multirow[t]{2}{*}{ TER } & $\begin{array}{l}\text { Correlation } \\
\text { Value }\end{array}$ & .037 & .044 & .308 & .251 & .014 & .037 & .048 & .003 & -.103 \\
\hline & $\begin{array}{l}\text { Sig. (2- } \\
\text { tailed) }\end{array}$ & .795 & .757 & .026 & .072 & .924 & .795 & .734 & .981 & .465 \\
\hline
\end{tabular}


3a. Is there significant difference in the ratings of the faculty based on the TER?

Summary of the Analysis of Variance for the TER by Department and by rater of the Respondents Tests of Between-Subject Effects

\begin{tabular}{|c|c|c|c|c|c|c|}
\hline \multicolumn{2}{|l|}{ source } & \multirow{2}{*}{$\begin{array}{c}\begin{array}{c}\text { Type III Sum } \\
\text { of Squares }\end{array} \\
31.174^{\mathrm{a}}\end{array}$} & Df & \multirow{2}{*}{$\begin{array}{r}\text { Mean } \\
\text { Square } \\
3.897\end{array}$} & \multirow[b]{2}{*}{2.878} & \multirow{2}{*}{$\begin{array}{l}\text { Sig. } \\
.012\end{array}$} \\
\hline Correct Model & Rater_stud & & 8 & & & \\
\hline & Rater_peer & $8.947^{b}$ & 8 & 1.118 & 3.682 & .002 \\
\hline & Rater_sup & $100.645^{c}$ & 8 & 12.581 & 5.902 & .000 \\
\hline \multirow[t]{3}{*}{ Intercept } & Rater_stud & 46778.784 & 1 & 46778.784 & 34544.547 & .000 \\
\hline & Rater_peer & 26705.623 & 1 & 26705.623 & 87913.852 & .000 \\
\hline & Rater_sup & 24757.246 & 1 & 24757.246 & 11613.986 & .000 \\
\hline \multirow[t]{3}{*}{.DeptCode } & Rater_stud & 31.174 & 8 & 3.897 & 2.878 & .012 \\
\hline & Rater_peer & 8.947 & 8 & 1.118 & 3.682 & .002 \\
\hline & Rater_sup & 100.645 & 8 & 12.581 & 5.902 & .000 \\
\hline \multirow[t]{3}{*}{ Error } & Rater_stud & 58.229 & 43 & 1.354 & & \\
\hline & Rater_peer & 13.062 & 43 & .304 & & \\
\hline & Rater_sup & 91.662 & 43 & 2.132 & & \\
\hline \multirow[t]{3}{*}{ Total } & Rater_stud & 75701.956 & 52 & & & \\
\hline & Rater_peer & 43468.537 & 52 & & & \\
\hline & Rater_sup & 40605.482 & 52 & & & \\
\hline \multirow[t]{3}{*}{ Correct Total } & Rater_stud & 89.403 & 51 & & & \\
\hline & Rater_peer & 22.009 & 51 & & & \\
\hline & Rater_sup & 192.307 & 51 & & & \\
\hline
\end{tabular}

a. $\mathrm{R}$ Squared $=.349$ (Adjusted R Squared $=.228$ )

b. $\mathrm{R}$ Squared $=.407$ (Adjusted R Squared $=.296)$

c. $\mathrm{R}$ Squared $=.523$ (Adjusted R Squared $=.435$ )

\section{Conclusions}

On the level of faculty efficiency as measured by TER, the teaching efficiency rating (TER) of MSU-TCTO faculty members is an aggregate of student's rating (30\%), peer's rating (30\%) and superior's rating (30\%) to get the total TER (Teaching Efficiency Rating). Thus, the sum of the three mean score which is 94.8960 is the MSU-TCTO faculty member average teaching efficiency rating or TER. It is also noted that the influence of socio-demographic profile of the respondents on the competencies of the faculty, the teaching efficiency rating (TER) is only significantly related to the respondents' status of appointment (Work status). While the rest of the variables for the demographic profile do not warrant for the significant relationship. However, on the significant difference in the ratings of faculty by department and by raters, there is a significant difference in the mean score of the faculty TER as rated by the students, peer, and superior by department.

Therefore, from the given result we cannot generalize the teaching competency of the faculty members. This is due to insufficient number of data presented, perhaps if data or sample size will be added then possibly we can warrant the teaching competency of the said study. 


\section{Recommendations}

From the given findings, here are some of the recommendations

1. More studies should be conducted related to teaching competency.

2. More respondents or bigger sample size must be considered.

3. Hiring of qualified and competent teachers is important.

4. Regular sending of faculty members to seminar/workshops and trainings.

5. Provision of adequate and updated materials for teaching.

\section{References}

[1] Agno, Lydia N. Principles of Teaching 2. A Modular Approach. Published in 2010 by C and E Publishing, Inc.

[2] Aquino, Gaudencio (1971). Curriculum Development Principles and Techniques.

[3] Adjawi, Maria Liza B. (2001). Teaching Efficiency Rating (TER) of the Muslim and Non-Muslim Faculty. Unpublished Thesis.

[4] Aradais, Langka B. (2000). Teaching effectiveness of the Public Secondary School Teachers of Bongao.

[5] Aripin, Hasana H. (2006). Professional Competencies and Instructional Needs of High School Teachers of vocational Schools in Tawi-Tawi: Implications to Quality Instruction.

[6] Barr (1961) conducted studies on "Professional Competency and Teacher Effectiveness".

[7] Bhagoliwal (1982) conducted a study entitled "A Study of Personality Characteristics associated with teaching effectiveness".

[8] Gupta R. C. (1976). Prediction of Teacher Effectiveness through Personality Tests".

[9] Kanakala, Jayaran (2010) conducted a study on "The Impact of Professional Competency and Creativity of Professional Pleasure".

[10] Karbasioun, M., Mlder, M., \& Biemeans, H. (2007). Competence Standards, Vocations and Professions: Competenceprofiling for Vocational and Professional Education.

[11] Madi, Marjuni M. (2005). Teachers' Performance and the National Achievement Test Results of Grade IV Pupils in the Division of Tawi-Tawi.

[12] Roy, Samanta (1971). "A study of Teacher Attitude and its relationship with Teaching Efficiency".

[13] Wesselink, R., Biemans, H., Mulder, M. \& Van den Elsen, E. R. (2007). Competitiveness Measurement of Curricula in Vocational Education: competence-Based Teaching and Learning. 